The inability of the Psammechinus miliaris H3 RNA to be processed in the Xenopus oocyte is associated with sequences distinct from those highly conserved amongst sea urchin histone RNAs

\title{
F.Schaufele and M.L.Birnstiel*
}

Institut für Molekularbiologie II, Universität Zürich, CH-8093 Hönggerberg, Zürich, Switzerland

Received July 10, 1987; Revised and Accepted September 10, 1987

\begin{abstract}
$3^{\prime}$ processing of precursors of the H3 RNA of the sea urchin Psammechinus miliaris in Xenopus oocytes is dependent upon sea urchin U7 snRNA. Sequences necessary for this interaction are highly conserved in all sea urchin histone precursor RNAs (including the Psammechinus H3) which, in contrast, are efficiently processed in the Xenopus oocyte without the addition of the homologous U7 snRNA. We resolve this seeming paradox by demonstrating here that the inability of the sea urchin Psammechinus miliaris H3 histone RNA to be processed in the Xenopus oocyte is associated with nucleotides immediately 3 ' to the conserved downstream sea urchin histone sequence element. Thus, a sequence-specific element (or lack of it) is responsible for the poor recognition of the Psammechinus H3 precursor RNA by the Xenopus processing machinery.
\end{abstract}

\section{INIRODUCTION}

The $3^{\prime}$ end of the mature histone message is formed through an endonucleolytic cleavage (1) of a precursor RNA in a reaction known as 3 ' processing $(2,3)$. This process had been best characterized for sea urchin histone RNAs. Sea urchin histone precursor RNAs contain two highly conserved sequence blocks flanking the site of the histone RNA mature $3^{\prime}$ end (4) both of which are important to $3^{\prime}$ processing $(5,6)$. The histone sequence upstream of the $3^{\prime}$ processing site is required as an RNA stem-loop structure specified by Watson-Crick base-pairing $(2,7)$ of which the primary sequence can be significantly altered without major consequences to histone RNA 3 ' processing in Xenopus oocytes or in sea urchin embryos $(7,8)$. The downstream conserved sea urchin histone RNA sequence element has no strict primary structure requirements except that it must be capable of forming Watson-Crick type base pairs (9) with a small nuclear RNA species (U7 snRNA) known to be required for sea urchin histone RNA $3^{\prime}$ processing (10). Thus, the sequences of the downstream sea urchin histone element and the $5^{\prime}$ end of the U7 SnRNA with which it base pairs are mutually dependent.

The upstream stem-loop structure is also highly conserved within verte- 
brate histone RNAs (4) and a "vertebrate-specific" sequence conservation can be detected extending a further 5 nucleotides upstream of this which is distinct from that observed in sea urchins (11). The downstream sequence element is conserved amongst vertebrate histone RNAs also as a sequence overlapping with (and extending 3' to) that observed for sea urchin histone RNAs $(12,13)$. The presence of both upstream and downstream mouse histone elements is necessary for efficient precursor RNA processing in vitro (0. Gick and M. L. B., unpublished data) and is sufficient to confer histone-specific RNA $3^{\prime}$ processing to RNA expressed from a chimaeric construct introduced into mouse cells (11) supporting the hypothesis that these histone RNA sequences may be retained in vertebrates (in modified form) since they are important to $3^{\prime}$ processing.

The observation that the sea urchin Psammechinus miliaris early histone H3 RNA is processed within the Xenopus oocyte very poorly unless supplied with a homologous sea urchin factor (14) led to the identification of U7 snRNA $(10,15)$. The inability of the Psammechinus H3 RNA to be processed in the Xenopus oocyte despite preserving the conserved sequence elements present in other sea urchin histone RNAs which are, in contrast, processed in the Xenopus oocyte has not been explained. It has been suggested that this sea urchin histone RNA may lack sequences required to interact with the Xenopus $3^{\prime}$ processing machinery (12). We demonstrate here that a histone H3 RNA transcribed from the gene of a different sea urchin species (Paracentrotus lividus) is correctly processed when injected into Xenopus oocytes. By exchanging sequences between the Paracentrotus and Psammechinus genes and resecting the region found to be functionally important, we localize the inability of the Psammechinus H3 RNA to be processed in the Xenopus oocyte to sequences lying immediately $3^{\prime}$ to the conserved sea urchin downstream element. The implications of this result with regards to any potential interactions with a putative Xenopus "U7-1ike snRNA" are discussed.

\section{MATBRTALS AND METHODS}

Plasmids were constructed by digesting the Psammechinus and Paracentrotus H3 genes at common restriction sites and replacing Psammechinus segments with the corresponding fragments from the Paracentrotus gene. In cases where the restrictions resulted in incompatible ends, fragments were first blunt-ended with the Klenow fragment of $\mathrm{E}$. coli polymerase: the ClaI site of the Psammechinus $\mathrm{H} 3$ gene was blunt-ended for ligating to the Paracentrotus ScaI site (constructs $\mathrm{H} 3 \mathrm{PmSPI}$ and $\mathrm{H} 3 \mathrm{PmCP} 1$ ) and, for the construction of the clones 
H3PmHfP1 and H3PmHf455P1, the HinfI sites of Paracentrotus H3 were blunt-ended for ligating to the Asp700 site within the clone pU-H3CAGA1-C (a derivative of the Psammechinus wild-type $\mathrm{H} 3$ gene containing two insertions--listed as lower case letters in the Psammechinus RNA sequence in Fig. 1 downstream of the conserved sea urchin histone sequence block--which constructed a new Asp700 site, see ref. 9). The Paracentrotus SacII-HinfI fragment was inserted between the SacII and EcoRI sites of PU-H3CAGA1-C to construct H3PmSP1Hf; a small deletion of 14 bp of Psammechinus sequence was incurred during the cloning of this construct but did not affect the interpretation of the results.

The 274 bp fragment of the clone H3PmHfP1 extending from the SacI site (position +260 in Fig. 1) to a Klenow polymerase-treated HinfI site (position +534) was cloned between the SacI and Klenow polymerase-blunt-ended EcoRI sites of the construct, 191093 (derivative of the Psammechinus H3 gene, ref. 6) to produce the clone H3PmHfP1-57. The same 274 bp fragment was also inserted into a SacI/SmaI digested pSP64 vector. Cutting this construct ( $\mathrm{Pr}$-H3HfP1274, not shown) at the BamHI site within the polylinker, followed by incubation with Ba131, T4 polymerase and BstEII (at position +351) in that order, resulted in the production of a series of fragments containing a common BstEII end but with variable amounts of DNA removed from the HinfI side. These fragments were inserted into EcoRI (Klenow treated)/BstEII digested 191093 to produce the clones, H3PmHfP1-31, -17 and -5 . Al1 these constructs contain Psammechinus sequences exactly as in $\mathrm{pU}-\mathrm{H} 3 \mathrm{wt}$ except for those nucleotides replaced by the Paracentrotus sequences shown white on black in Fig. $4 \mathrm{~b}$.

$1 \mathrm{ng}$ of each clone was microinjected per Xenopus oocyte and RNA was harvested the next day (see ref. 19). In the experiments shown in Figs. 3 and 4, 2 ng of either U2/U7 or pUC18 were coinjected with the H3 test clones; functional sea urchin U7 snRNA is transcribed from the U2/U7 clone within the Xenopus oocyte (17).

All S1 mappings were done with a $5^{\prime}$ probe labelled at the HpaII site (position +99 in Fig. 1) which protected a fragment of 154 nucleotides. 3' probes were labelled at either a BstEII (Fig. 2) or TaqI site (Figs. 3 and 4) protecting properly $3^{\prime}$ processed RNAs of 113 or 77 nucleotides, respectively.

\section{RESULTS AND DISCUSSION}

Experimental Rationale

Transcripts of the Paracentrotus lividus H3 gene produced either after injection into Xenopus oocytes or in vitro from a bacterial promoter are processed in the Xenopus oocyte (F. S., unpublished data) whereas similarly 


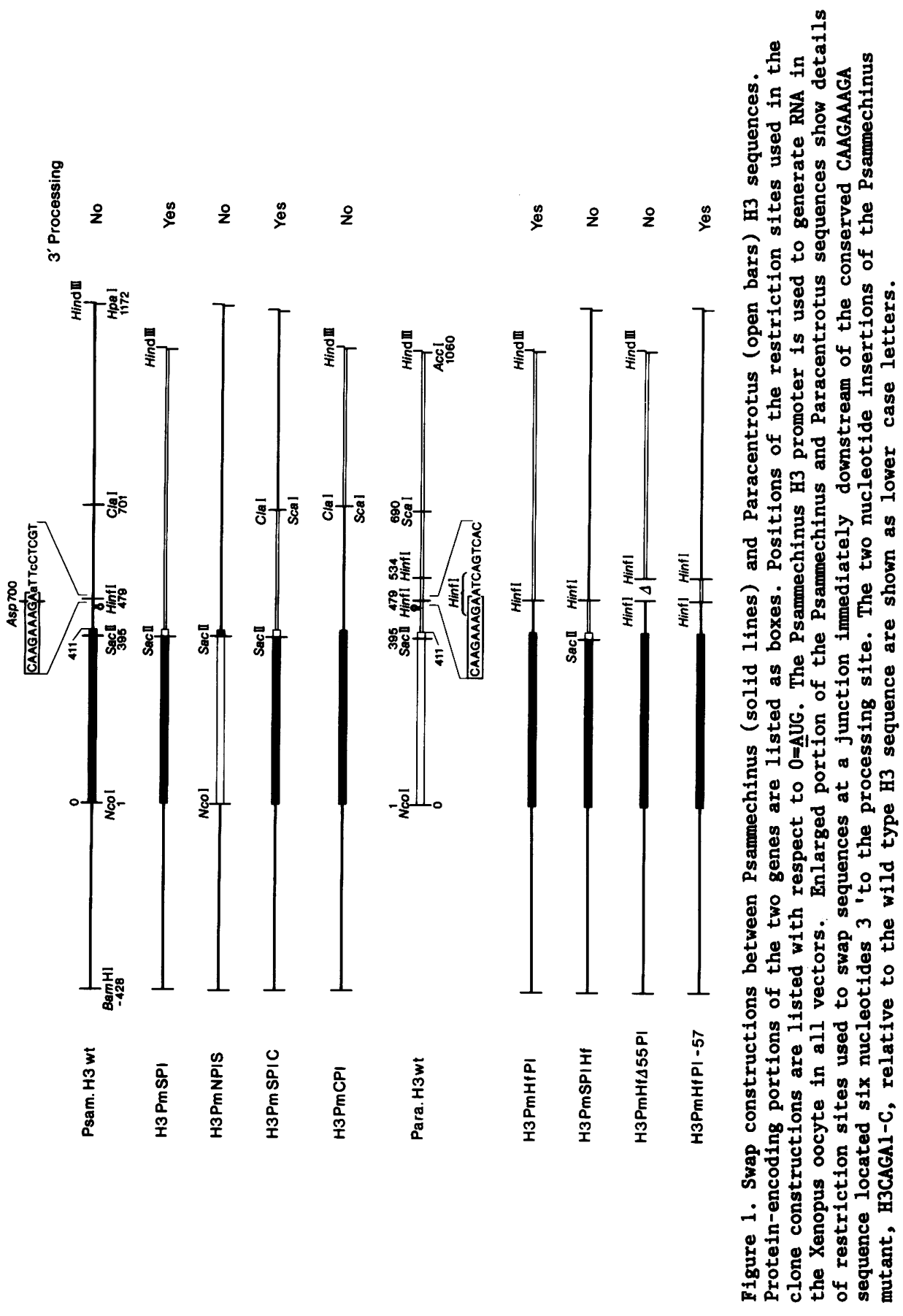




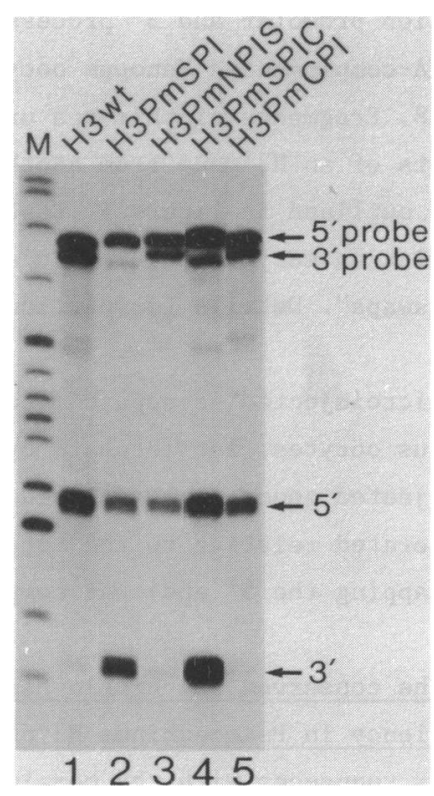

Figure 2. S1 analysis of RNA generated from the indicated constructs following microinjection into Xenopus oocytes. $5^{\prime}, \mathrm{H} 3$ transcripts properly initiated from the H3 promoter. $3^{\prime}$, correctly processed H3 RNAs. $5^{\prime}$ or $3^{\prime}$ probe, input probes used for the $5^{\prime}$ or $3^{\prime}$ maps. Differences in the size of the input $3^{\prime}$ probes reflects the differences in the cognate probes used for each mapping. M, pBR322 HpaII-digested size markers.

synthesized transcripts of the Psammechinus miliaris H3 gene are processed only after coinjection into the same oocytes of a small RNA fraction containing U7 snRNA $(2,18)$. Both species contain the highly conserved sequence elements common to sea urchin histone RNAs (4) which have been shown to be important for their $3^{\prime}$ processing $(2,5-7,9)$. Thus, it seemed probable that these sequence elements were not responsible for the inactivity of the Psammechinus H3 RNA (or for the relative activity of the Paracentrotus H3 RNA) in the Xenopus oocyte $3^{\prime}$ processing system implicating an, as of yet, unknown principle. We have therefore analysed which sequence could be contributing to this phenomenon by the systematic exchange of segments of the Psammechinus and Paracentrotus genes followed by a more precise resection analysis within the region identified to be important.

Construction of the clones used in the following experiments is outlined in figure 1. DNAs injected into Xenopus oocytes are derivatives of pU-H3wt (see ref. 9), a 1600 bp BamHI to HpaI fragment of the Psammechinus miliaris H3 gene 
including both transcription promoter and $3^{\prime}$ processing signals (effective only in sea urchins or U7 snRNA-complemented Xenopus oocytes) inserted into BamHI/HindII-digested pUC8. Pragments of this sea urchin H3 gene were deleted and replaced with fragments of an $\mathrm{H} 3$ gene from another sea urchin species, Paracentrotus lividus, as outlined in figure 1. Restriction enzyme sites common to both $\mathrm{H} 3$ genes or restriction enzymes cutting in analogous positions were used to make these gene "swaps". Details for particular clones are described in Materials and Methods.

H3 constructs were microinjected as supercoiled DNA into the germinal vesicle of stage VI Xenopus oocytes, let incubate overnight and the transcripts produced from the microinjected genes were analysed by S1 mapping; the amount of histone $\mathrm{H} 3^{\prime}$ ' ends generated relative to the total number of $\mathrm{H} 3$ transcripts made (as detected by S1 mapping the $5^{\prime}$ end) was compared (see Figs. 2, 3 and 4).

Sequences downstream of the conserved sea urchin histone sequences are responsible for the deficiency in Psammechinus $\mathrm{H} 3$ processing

Replacing Psammechinus sequences with the complete 3' trailer and 609 nucleotides downstream of the mature $3^{\prime}$ end of the Paracentrotus gene resulted in the efficient production of $3^{\prime}$ ends of RNAs transcribed from this construct (H3PmSP1) in the absence of sea urchin U7 snRNA (Fig. 2, lane 2). In contrast, transcripts generated from the wild type Psammechinus $\mathrm{H} 3$ gene were processed very inefficiently (Fig. 2, lane 1). Replacing almost the entire coding body of the Psammechinus gene with Paracentrotus sequences did not significantly rescue the ability of these transcripts to be processed in the absence of sea urchin U7 snRNA (Fig. 2, lane 3, H3NP1S). Thus, some sequence within the 3 ' non-coding trailer or within the downstream spacer sequences of the Paracentrotus H3 RNA was capable of conferring on the Psammechinus H3 RNA the ability to be processed by the Xenopus $3^{\prime}$ processing machinery (the converse argument, that some deleterious Psammechinus sequence was removed will be discussed later). This sequence was further delimited to the 3 ' non-coding trailer and 211 nucleotides of Paracentrotus sequence downstream of the processing site since RNAs containing the Paracentrotus SacII-ScaI fragment (replacing the Psammechinus SacII-ClaI fragment) were processed in the Xenopus oocyte ( $\mathrm{Fig}$. 2, lane 4, H3PmSP1C) whereas a construct containing Paracentrotus H3 sequences only downstream of the ClaI/ScaI site was not (Fig. 2, lane 5, H3PmCP1).

Replacing 295 nucleotides of Psammechinus H3 RNA sequences encompassing both the upstream (stem-1oop) and downstream histone-specific consensus blocks 


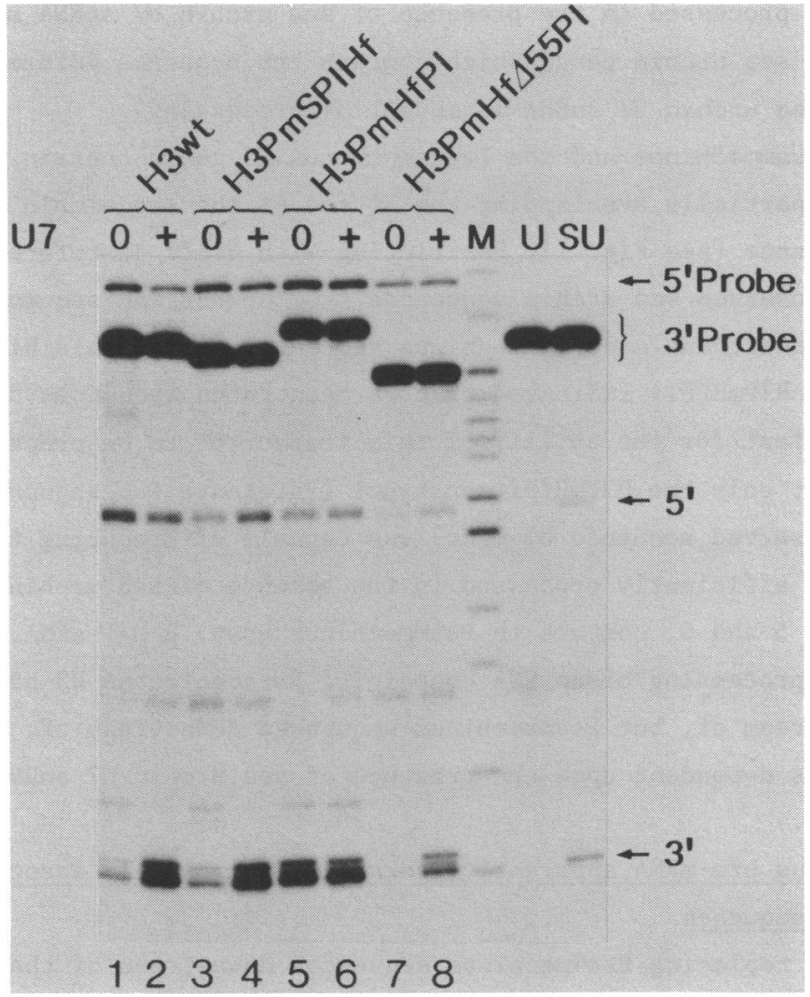

Figure 3. S1 analysis of RNA generated from the indicated constructs following microinjection into Xenopus oocytes with $(+)$ or without $(0)$ coinjection of a vector expressing U7 snRNA. Symbols are as described in Fig. 2. U, negative control S1 mapping with uninjected Xenopus oocyte RNA. SU, positive control S1 mapping with $20 \mathrm{ng}$ of Psammechinus blastula RNA and H3 wild type probe.

with the corresponding Paracentrotus sequences was therefore sufficient to allow this RNA to be processed efficiently in Xenopus oocytes. We next sought to determine the relationship of the two highly conserved sea urchin sequence blocks to the inability of the Psammechinus H3 RNA to be processed by the Xenopus oocyte. New constructs were made and tested either with or without the coinjection of U2/U7wt (see ref. 9), a plasmid in which a Xenopus U2 snRNA promoter is used to drive the expression of the sea urchin U7 snRNA in Xenopus oocytes (17). Efficient $3^{\prime}$ end formation in the absence of sea urchin U7 snRNA ( 0 U7, Fig. 3) and the inability of sea urchin U7 snRNA to further enhance 3' processing activity ( $+U 7)$ demonstrates the ability of a given construct to be recognized and processed by the Xenopus $3^{\prime}$ processing machinery. Logically, all 
constructs are processed in the presence of sea urchin U7 snRNA since they are derivatives of sea urchin genes which contain the sequence information required for sea urchin U7 snRNA-dependent $3^{\prime}$ processing.

Both the Psammechinus and the Paracentrotus $\mathrm{H} 3$ genes contain a HinfI restriction site partially overlapping the $3^{\prime}$ end of the sea urchin downstream consensus sequence (see Fig. 1). Restricting with HinfI therefore serves to dissect the conserved sea urchin sequences from downstream sequences. Such a "swap" of Paracentrotus and Psammechinus sequences around this HinfI site (H3PmSP1Hf and H3PmHfP1) indicated that Paracentrotus sequences $3^{\prime}$ to the HinfI site are important for the ability of this transcript to be processed in the Xenopus oocyte; only the H3PmHfP1 construct (Paracentrotus sequences 3 ' to the sea urchin conserved sequence element) was capable of producing transcripts which could be efficiently processed in the absence of sea urchin U7 snRNA (Fig. 3, lanes 5 and 6, compare to Psammechinus H3wt, 0 U7 snRNA, lane 1). In constrast, $3^{\prime}$ processing of an RNA containing Paracentrotus $\mathrm{H} 3$ histone sequences upstream of, but Psammechinus sequences downstream of, the HinfI site (H3PmSP1Hf) was dependent upon the presence of sea urchin U7 snRNA (Fig. 3, lanes 3 and 4).

The Psammechinus pre-mRNA apparently lacks a signal closely associated with the CAAGAAAGA sequence

Therefore, replacing Psammechinus sequences downstream of the sea urchin conserved sequence blocks with the corresponding fragment of the Paracentrotus gene resulted in the efficient $3^{\prime}$ processing in the Xenopus oocyte of the otherwise poorly processed Psammechinus RNA. This "rescue" of 3 ' processing could formally be the result of one of two scenarios: (i) an RNA sequence within the Psammechinus transcript somehow inhibitory to $3^{\prime}$ processing activity was removed and replaced by rather inert Paracentrotus sequences or (ii) the Paracentrotus fragment actively participates in processing rescue by supplying a sequence, absent from the Psammechinus RNA, which is required for $3^{\prime}$ processing in the Xenopus oocyte. The observation that deleting a small (55 nucleotide) fragment of Paracentrotus sequence from the H3PmHfP1 construct resulted in the loss of $3^{\prime}$ processing activity (Fig. 3, lanes 7 and 8, H3PmHf 455 P1) supports the hypothesis of an auxiliary required sequence element for efficient $3^{\prime}$ processing in the Xenopus oocyte. Note, however, that we cannot categorically dismiss the possibility that the 55 nucleotide deletion resulted in the acquisition of another sequence inhibitory to $3^{\prime}$ processing.

Thus, the ability of the H3PmHfP1 RNA to be $3^{\prime}$ processed in the Xenopus 
a

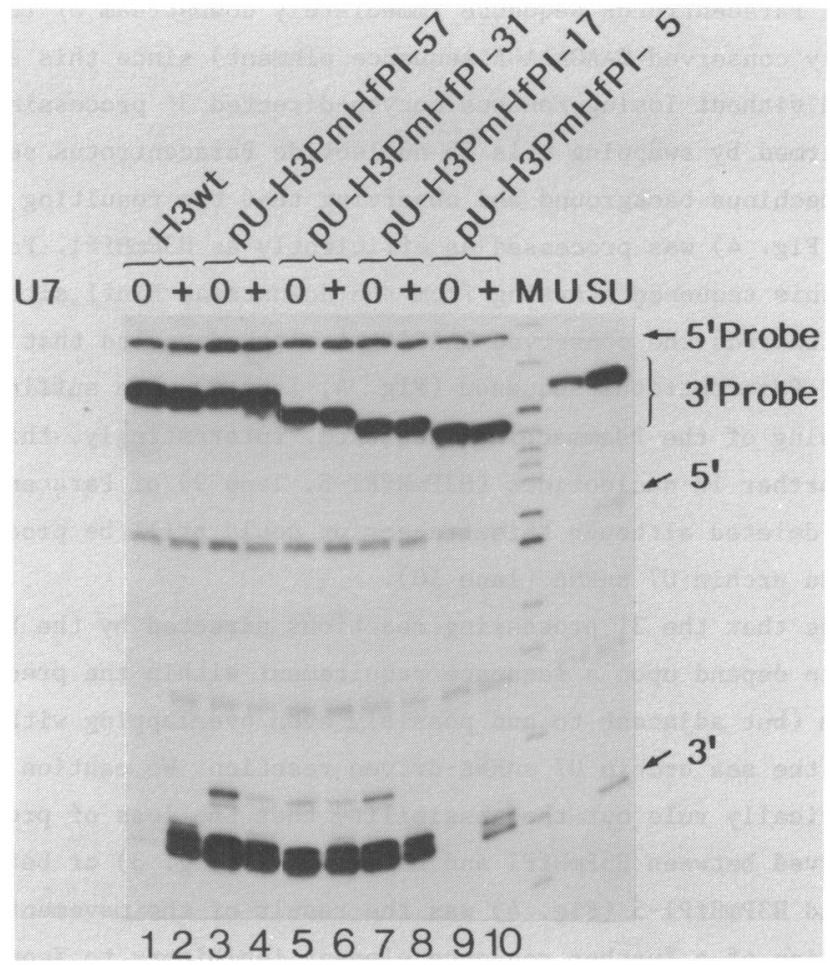

b

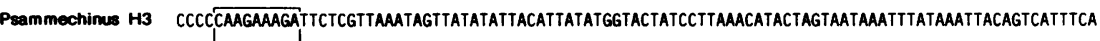

Paracentrotus $\mathrm{H3}$

DU-H3PmHfPI-57

DU-H3PmHFPI-31

DU-H3PMHFPI-17

DU-H3PmHFPI- 5

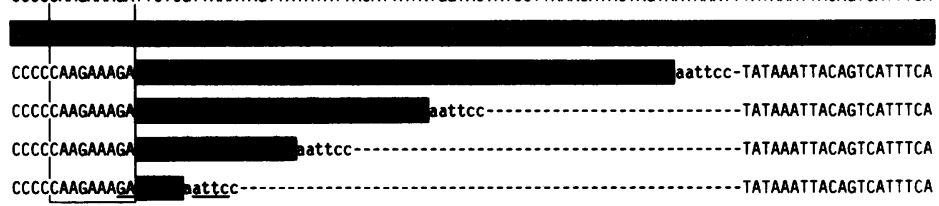

$-U 7 \quad+U 7$

Figure 4. a, S1 mappings of RNAs generated from the indicated constructs following microinjection into Xenopus oocytes. Symbols are as described in Figs. 2 and 3 . b, Sequences downstream of the conserved CAAGAAAGA element (boxed) of the constructs analysed in Fig. 4a. Paracentrotus H3 sequences are shown white on black. Remnants of the EcoRI linker derived the from clone 191 093 are shown as lower case letters. -, nucleotides deleted relative to the wild type constructs. Processing ( + ) or not ( 0$)$ of RAAs transcribed from the injected genes in the absence (-U7) or presence ( $+U 7$ ) of a construct expressing the sea urchin U7 snRNA is indicated. A potential secondary structure created in pU-H3PmHfP1-5 is underlined. 
oocyte probably resided at least partially (if not totally) within the first 55 nucleotides of Paracentrotus sequence immediately downstream of the HinfI site (and the highly conserved CAAGAAAGA sequence element) since this sequence could not be deleted without losing Xenopus oocyte-directed 3 ' processing activity. This was confirmed by swapping this 55 nucleotide Paracentrotus sequence alone into the Psammechinus background and observing that the resulting transcript (H3PmHfP1-57, Fig. 4) was processed as efficiently as H3PmHfP1. Furthermore, deletions of this sequence starting from the downstream HinfI site extending progressively towards the conserved CAAGAAAGA block revealed that 17 nucleotides of Paracentrotus sequence (Fig. 4, lane 7) were sufficient to drive the $3^{\prime}$ processing of the Psammechinus sequence. Interestingly, this ability was lost once a further 12 nucleotides (H3PmHfP1-5, lane 9) of Paracentrotus sequence were deleted although this transcript could still be processed in the presence of sea urchin U7 snRNA (lane 10).

We conclude that the $3^{\prime}$ processing reactions directed by the Xenopus oocyte seems to depend upon a sequence requirement within the precursor RNA different from (but adjacent to and possibly even overlapping with) that necessary for the sea urchin U7 snRNA-driven reaction. We caution again that we cannot categorically rule out the possibility that the loss of processing activity observed between $\mathrm{H} 3 \mathrm{PmHfP} 1$ and H3PmHf455P1 (Fig. 3) or between H3PmHfP1-17 and H3PmHfP1-5 (Fig. 4) was the result of the movement into a critical location of a further sequence element inhibitory to Xenopus-directed histone RNA 3 ' processing. However, such an explanation requires the influence of at least three independently positioned inhibitory sequences (one within the Psammechinus transcript, another within the Paracentrotus sequence or formed at the junction of the H3PmHf $455 \mathrm{P} 1$ deletion, and another Psammechinus sequence further downstream which exerts it effect only within the H3PmHfP1-5 RNA). In addition, none of these hypothesized inhibitory sequences apparently hindered the ability of the sea urchin U7 snRNA to recognize its target within the adjacent GAAAGA sequence. Certainly the most straight forward argument would be that the Paracentrotus H3 RNA contained within its sequence an element required to be present for efficient $3^{\prime}$ processing in Xenopus oocytes lying outside of the sea urchin conserved sequence block. The $3^{\prime}$ border of this element seems to lie between 5 and 17 nucleotides of Paracentrotus sequence downstream of the conserved CAAGAAAGA block. However, it could still be possible that the $3^{\prime}$ ' boundary of this element may be located even closer to the conserved sequence element since a potential secondary structure, formed at the junction of the H3PmHfPI-5 deletion (see Fig. 4), may have affected the 
interpretation of these experiments. Nevertheless, it is clear that the sea urchin U7 snRNA-independent processing reaction in the Xenopus oocyte depends upon a sequence immediately downstream of the CAAGAAAGA block which is not absolutely essential to U7 snRNA-driven $3^{\prime}$ processing. This sequence element would be obviously species(Xenopus)-specific since its absence from the Psammechinus H3 RNA does not affect the ability of this RNA to be processed correctly and efficiently within the sea urchin (9).

Implications for a Xenopus U7-1ike snRNA

The location of the additional sequence requirement for histone RNA 3 ' processing directed solely by the Xenopus oocyte is intriguing in light of our current understanding of the $3^{\prime}$ processing reaction. The downstream sequence block of the sea urchin histone RNA is functionally recognized by the sea urchin U7 snRNA only when the two RNAs retain base complementarities (9). A corresponding sequence possibly necessary for histone $3^{\prime}$ processing in a vertebrate test system extending further downstream than its sea urchin counterpart has been proposed (12). The data presented here and an analysis of the sequence requirements for efficient mouse histone $\mathrm{H} 4$ processing in mouse cells (11) or HeLa extracts (0. Gick and M. L. B., unpublished data) support this concept although, in our case, the required sequences seem to extend a few nucleotides further downstream than previously predicted (12). Comparing RNA sequences similarly located with respect to the processing site of those RNA species known to be processed in the Xenopus oocyte reveals some weak conservation of the first seven nucleotides downstream of the polypurine tract (CAAGAAGA in sea urchins; F. S. and M. L. B., unpublished data); however, the corresponding Psammechinus $\mathrm{H} 3$ sequences also do not differ striking1y from this weak consensus so it is not clear from such comparisons how this RNA should be excluded from being processed in the Xenopus oocyte although it is tempting to propose that subtle differences in this region could be involved. Additionally, RNA transcribed from a Psammechinus H2A gene can be effectively processed in Xenopus oocytes when containing only 9 nucleotides of H2A sequence downstream of the CAAGAAAGA element (7).

However, the vertebrate consensus sequence (12, updated in ref. 13) is quite diffuse, being variable at almost every position. Moreover, many of the sea urchin histone sequences (including the Paracentrotus sequences presented here) do not fit very well to the downstream portions of the proposed vertebrate consensus sequence but are nevertheless processed in the Xenopus oocyte although at least the Psammechinus H2A and H2B RNAs are processed much less rapidly than a Xenopus H4 RNA (19). If a Xenopus U7-1ike RNA exists which 
functions similarly to its sea urchin counterpart, it is possible that it may possess functionally relevant base complementarities further downstream of the polypurine stretch but such a base pairing would either not have to involve contacts at $100 \%$ of the sequences or have to be mediated by multiple snRNA entities in order to account for the wide variation in histone RNA sequences which are recognized. Alternatively, the weak conservation of this sequence may also indicate that the function of the sea urchin U7 snRNA may be taken over in Xenopus by a less sequence-specific interaction, such as could be mediated by a protein. Some combination of RNA and protein-directed recognition of the downstream element may similarly also be possible.

Whatever the nature of the interaction, we have presented evidence here that histone RNA $3^{\prime}$ maturation by the endogenous Xenopus processing machinery involves a cis-acting sequence distinct from that required in the sea urchin or in sea urchin U7 RNA-complemented Xenopus oocytes. Thus, the paradoxical processing deficiency of the Psammechinus H3 RNA has been explained as a consequence of this species-specific sequence requirement.

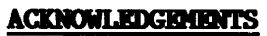

We wish to thank Chris Hug for her excellent technical help, Fritz Ochsenbein for his preparation of the figures and Dani Schümperli and Oki Gick for critical reading of the manuscript. This work was in part-fulfillment of the Ph.D. requirements for F. S. and was supported by the Kanton Zürich and the Swiss National Science Foundation. F. S. was partially supported by a postgraduate scholarship from the Natural Sciences and Engineering Research Council of Canada.

*To whom correspondence should be addressed at: Institut für Molekularpathologie, Himmelpfortgasse 1/3, A-1010 Vienna, Austria

\section{RRTERTings}

1. Gick, 0., Krämer, A., Keller, W. and Birnstie1, M. L. 1986 EMBO J. 5, 1319-1326.

2. Birchmeier, C., Schümper1i, D. Sconzo, G. and Birnstiel, M. L. 1984. Proc. Nat1. Acad. Sci. USA 81,1057-1061.

3. Krieg, P. A. and Melton, D. A. 1984. Nature 308, 203-206.

4. Hentschel, C. C. and Birnstiel, M. L. 1981. Ce11 25, 301-313.

5. Birchmeier, C., Grossched1, R. and Birnstiel, M. L. 1982. Ce11 28, 739-745.

6. Georgiev, O. and Birnstiel, M. L. 1985. EMBO J. 4, 481-489.

7. Birchmeier, C., Folk, W. and Birnstiel, M. L. 1983. Cel1 35, 433-440.

8. Schaufele, F. and Birnstiel, M. L., submitted.

9. Schaufele, F., Gilmartin, G. M., Bannwarth, W. and Birnstie1, M. L. 1986. Nature 323, 777-781. 
10. Strub, K., Galli, G., Busslinger, M. and Birnstiel, M. L. 1984. EMBO J. 3, 2801-2807.

11. Stauber, C., Lüscher, B., Eckner, R., Lötscher, E. and Schümperli, D. 1986. EMBO J. 5, 3297-3303.

12. Turner, P. C., Aldridge, T. C., Woodland, H. R. and 01d, R. W. 1983. Nucleic Acids Res. 11, 4093-4107.

13. Birnstiel, M. L., Busslinger, M. and Strub, K. 1985. Cell 41, 349-359.

14. Stunnenberg, H. G. and Birnstiel, M. L. 1982. Proc. Nat1. Acad. Sci. USA 79, 6201-6204.

15. Galli, G., Hofstetter, H., Stunnenberg, H. G. and Birnstiel, M. L. 1983. Cel1 34, 823-828.

16. Probst, E., Kressman, A. and Birnstiel, M. L. 1979. J. Mol. Biol. 135, 709-732.

17. Strub, K. and Birnstiel, M. L. 1986. EMBO J. 5, 1675-1682.

18. Hentsche1, C. C., Probst, E. and Birnstie1, M. L. 1980. Nature 288, 100-102.

19. Georgiev, O., Mous, J. and Birnstiel, M. L. 1984. Nucleic Acids Res. $12,8539-8551$. 